\title{
Symposium on the uses and control of the newer antibacterial agents ${ }^{1}$
}

There are now a very large number of antibacterial agents from which a choice must be made when treating patients with infection. The sheer number of antibacterial agents has made this choice not always an easy one.

For simplicity the antibacterial agents may be arranged in four groups as shown in Table I, which has been adapted from a similar table first published by the late Professor Mary Barber in 1966, brought up to date by Eykyn and Phillips in 1969, and finally revised here.

The greatest advance in antimicrobial therapy that has occurred since the début of benzylpenicillin was undoubtedly the introduction, beginning in 1960, of the semisynthetic penicillins but still referred to as the 'new penicillins'.

Today we wish to review three groups of drugs introduced even more recently. First, rifampicin and clindamycin ( 7 chlorlincomycin), then the cephalosporins, and finally, sulphamethoxazole/trimethoprim (Bactrim or Septrin).

MARK RIDLEY

Chairman

\begin{tabular}{|c|c|c|c|c|}
\hline $\begin{array}{l}\text { Group 1: } \\
\text { For Gram-positive } \\
\text { Bacteria and } \\
\text { Gram-negative Cocci }\end{array}$ & $\begin{array}{l}\text { Group 2: } \\
\text { For Gram-negative } \\
\text { Bacilli }\end{array}$ & $\begin{array}{l}\text { Group 3: } \\
\text { Broad Spectrum }\end{array}$ & $\begin{array}{l}\text { Group 4: } \\
\text { For Urinary } \\
\text { Infections Only }\end{array}$ & $\begin{array}{l}\text { Group 5: } \\
\text { For Tuberculosis }\end{array}$ \\
\hline $\begin{array}{l}\text { Penicillins: } \\
\text { Benzylpenicillin } \\
\text { Phenoxymethyl- } \\
\text { penicillin } \\
\text { Phenethicillin } \\
\text { Methicillin } \\
\text { Cloxacillin } \\
\text { Flucloxacillin } \\
\text { Erythromycin } \\
\text { Lincomycin }{ }^{1} \\
\text { Vancomycin } \\
\text { Novobiocin } \\
\text { Fucidin } \\
\text { Rifamycins: } \\
\quad(\rightarrow 2,5) \\
\text { Rifamide } \\
\text { Rifampicin }\end{array}$ & $\begin{array}{l}\text { Streptomycin } \\
\text { Neomycin } \\
\text { Kanamycin } \\
\text { Gentamicin } \\
\text { Penicillins: } \\
\text { ampicillin }(\rightarrow 1) \\
\text { carbenicillin }(\rightarrow 1)\end{array}$ & $\begin{array}{l}\text { Cephalosporins: } \\
\text { cephaloridine } \\
\text { cephalothin } \\
\text { cephalexin } \\
\text { Sulphonamides } \\
\text { Trimethoprim- } \\
\text { sulphamethoxazole }\end{array}$ & $\begin{array}{l}\text { Nitrofurantoin } \\
\text { Nalidixic acid }\end{array}$ & $\begin{array}{l}\text { Streptomycin } \\
\text { PAS } \\
\text { Isoniazid } \\
\text { Thiacetazone } \\
\text { Ethionamide }\end{array}$ \\
\hline
\end{tabular}

An arrow $\rightarrow$ indicates that an antibiotic may also be useful for some organisms in the group indicated.

${ }^{1}$ Lincomycin should not be used against Gram-negative cocci.

References

Barber, M. (1966). In Therapeutic Uses of Antibiotics in Hospital Practice, p. 203, edited by M. Ridley and I. Phillips. E. and S. Livingstone, Edinburgh and London.

Eykyn, S., and Phillips, I. (1969). Advances in Antibiotics. The Practitioner, 203, 501.

${ }^{1}$ This symposium was held in September 1970 at the autumn meeting of the Association of Clinical Pathologists. 